\title{
Expression and purification of the Green Fluorescent Protein via immobilized metal ion affinity.
}

\author{
Olga Dagmara Sieluzycka ${ }^{1}$ \\ ${ }^{1}$ Queen Mary University of London
}

April 28, 2020

Expression and purification of the Green Fluorescent Protein via immobilized metal ion affinity.

Olga D. Sieluzycka

School of Biological and Chemical Sciences (Fogg Building)

Queen Mary University of London Mile End Road

London E14NS UK

ABSTRACT: The aim of the experiment is to express and purify recombinant His-tagged Green Fluorescent Protein (GFP) protein inEscherichia coli (E.coli) using metal affinity chromatography. GFP is an excellent trafficking tool in modern biochemistry. The protein was expressed in E.Coli and introduced to the required organism by a plasmid transformation. Plasmid containing the GFP was controlled by the T7 promoter had ampicillin resistance and had an N-terminal His6 tag fusion to facilitate the process of purification in the later stages of experiment. The expression of proteins was confirmed by the SDS gel by comparing the bands widths. The goal of experiment was not fully met.

Introduction: The main objective of the experiment is to express and purify recombinant His tagged, Green Fluorescent Protein (GFP). GFP is a protein native to a jellyfish, Aequorea Victoriaand due to its remarkable fluorescence it has a vast amount of applications in modern biochemistry such as in vivo labelling and visualization of cells structures. Since the GFPs fluorescence disturbed by unfavorable conditions can recover in the correct $\mathrm{pH}$ and temperature it is incredibly useful in molecular biology and its ability to track "real time" changes in cells with a "naked eye" captured the attention of various researchers. (Wu et al., 2008) Another big advantage of using GFP over other molecular trackers is that it does not require any substrates or cofactors has a low toxicity towards examined cells and does not alter the localization of its fusion partner. (Kumar et al., 2016)

GFP, presented by figure 1A, is a 238 amino acid protein that adapts a beta barrel fold. This particular structure, containing a chromophore is responsible for its fluorescent properties and if the fold is altered the fluorescence is lost. Given the usefulness of GFP in various aspects of molecular biology, a number of different protocols for its expression and purification have been designed. It is a relatively easy and stable protein to purify, hence being treated as a way of learning techniques and methods used in modern protein cloning and obtaining recombinant proteins. GFP chosen for the following experiment has a monomeric structure and possesses one mutation. E. Coli is an organism chosen for the expression of the recombinant GFP. This bacteria is one of the most common choices for the recombinant proteins production since it has very high and effective growth kinetics, doubling its amount in just 20 minutes. The bacteria's optimal growth is at 37 degrees Celsius in glucose and salt media, which is relatively cheap and easy to provide, making the E.Coli the appropriate organism suitable for the experiment. 


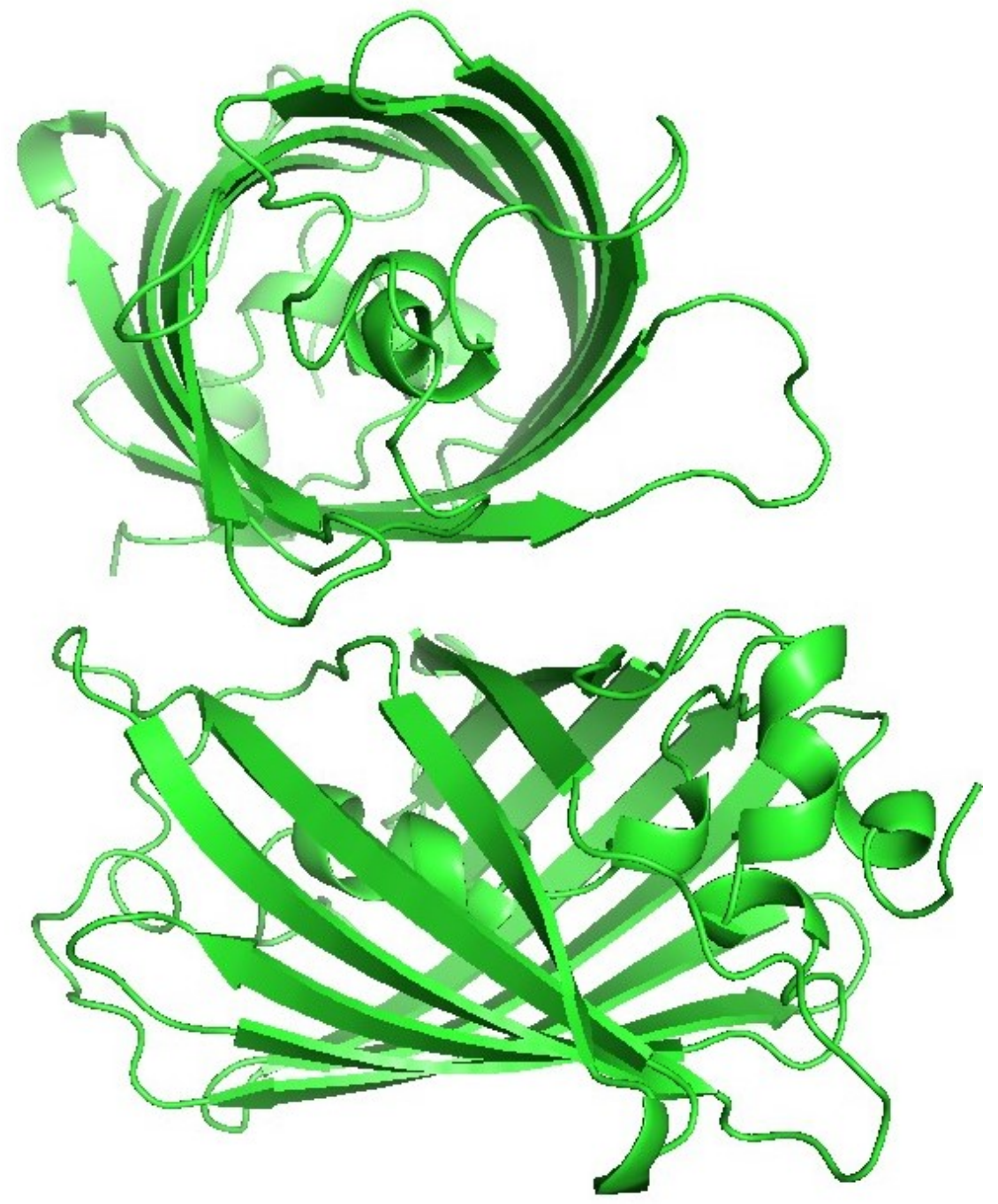

Figure 1: 3D-GFP monomer structure in its native beta barrel fold generated in PyMol. The structure consists of 11-stranded barrel and a central helix.

Due to the use of polyhistidine-tags (His tags), the GFPs affinity for divalent metal ions, such as Ni2+ increases greatly which enables the efficient and effective use of Immobilized Metal Affinity Chromatography (IMAC) which is a convenient method of purification but cannot be implemented without the use of his tags.

Materials and Methods: 50ul of chemically competent E.Coli (Bl21D3) were used in the experiment. The cells were incubated with pRSET vector, presented by figure 2, containing both GFP insert and ampicillin resistance gene. After thawing a sample on ice to ensure a plasmid intake, transduced BL21D3 mixed with Lysogeny broth (LB) were incubated at 370 for 60 minutes. Following the incubation 20 and $200 \mathrm{ul}$ of sample was introduced on 2 halves of ampicillin enriched Agar plate, figure 3A. The plates were incubated overnight at 170. Following the incubation, a colony was selected and ejected into $10 \mathrm{ml}$ of LB broth with $100 \mathrm{ug} / \mathrm{ml}$ ampicillin to create a starter culture. The culture was incubated overnight on a shaker, to prevent cells form settling. To create a lager culture, $1 \mathrm{ml}$ of starter culture and $100 \mathrm{ml}$ of $100 \mathrm{ug} / \mathrm{ml}$ ampicillin were introduced 


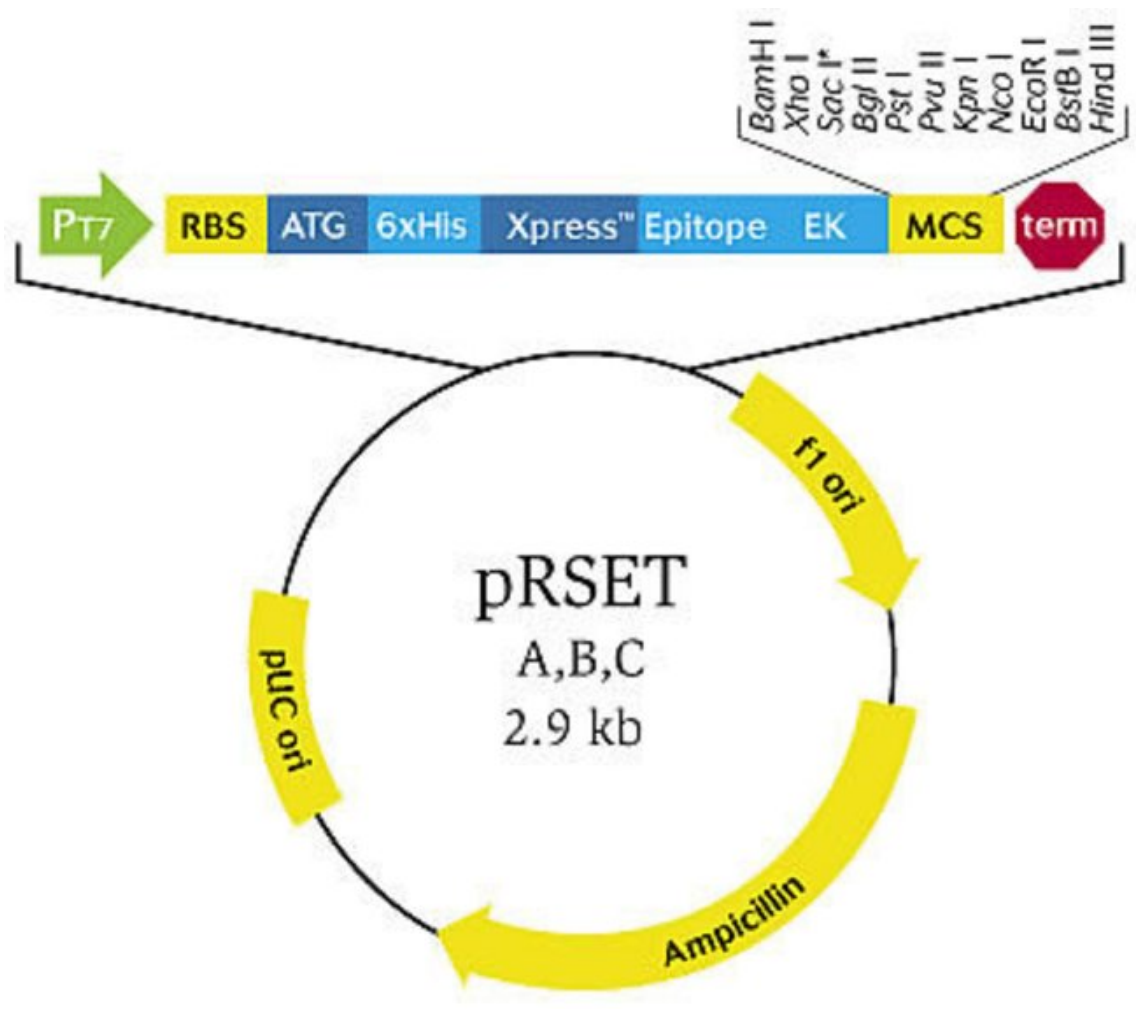

Figure 2: pRSET vector used in the experiment, designed for high level of prokaryotic expression, controlled by a T7 promoter. (Assets.thermofisher.com, 2018)

to $100 \mathrm{ml}$ of $2 \mathrm{XYT}$ and incubated in 37 0C shaker for 6 hours, to reach the OD600. $50 \mathrm{ul}$ of "pre induced" sample were taken from the culture and injected into an Eppendorf tube. The remaining culture was induced by adding 50ul of IPTG to force the production of rGFP. After the induction the culture was incubated overnight in 370C shaker. Sample of $50 \mathrm{ul}$ of the post induction sample was injected into an Eppendorf. Cells expressing rGFP were harvested by centrifugation and the pellet was weighted and re-suspended in adequate amount of BugBuster TM ( $5 \mathrm{ml}$ of BugBuster per each gram of pellet paste). Following the re-suspension the pellets were centrifuged again at $13 \mathrm{krpm}$ for 20 minutes. After the centrifugation a sample of supernatant was conserved. The pellets were re-suspended. A Ni2+ chromatographic column was equilibrated using $10 \mathrm{ml}$ of wash buffer (phosphate buffer, pH $820 \mathrm{mM}$ imidazole, $300 \mathrm{mM} \mathrm{NaCl}$ ). A sample of supernatant and flow through were collected. The column was washed with $4 \mathrm{ml}$ of elution buffer (phosphate buffer, pH8, $250 \mathrm{mM}$ imidazole, $300 \mathrm{mM} \mathrm{NaCl}$ ) and elute was collected. SDS-PAGE was runned at $100 \mathrm{~V}$ for 2 hours. The wells were loaded with 10ul of sample and the Laemil buffer. The gel was stained overnight and examined on a following day.

Results: The goal of the experiment was not fully met. Despite performing a successful plasmid transformation, confirmed by the cellular growth on the antibiotic containing media, the final SDS-PAGE measurement failed to show a decisive evidence of the GFP presence. In this experiment the particular E.Coli strain used, the pRSET vector is repressing the promoter region. The promoter is essential for the polymerase (T7) to work. The binding of $\mathrm{T} 7$ polymerase to the promoters of the vector, triggers the polyhistidine tags that express the GFP fluorescence. This enables a low production of rGFP however it is not enough to see a clear purification band on the SDS-PAGE, hence the IPTG induction takes place that stops the repressor from working. As a result, an increased production of GFP should be observed. 
The first time an undesirable outcome of the experiment was noticed was after the 6 hour incubation period at $370 \mathrm{C}$ before the bacterial inoculation. The culture should have reached the chosen optical density, OD600, with the liquid being cloudy. At this optical density, the bacteria should still be healthy, in their growing phase, large in numbers. The observed solution was yellowish brown as expected but completely clear which indicated that the chosen optical density was not reached. A number of colleagues had the same issue with their cultures however the bacteria had to be induced regardless of the desiarable OD not being reached.

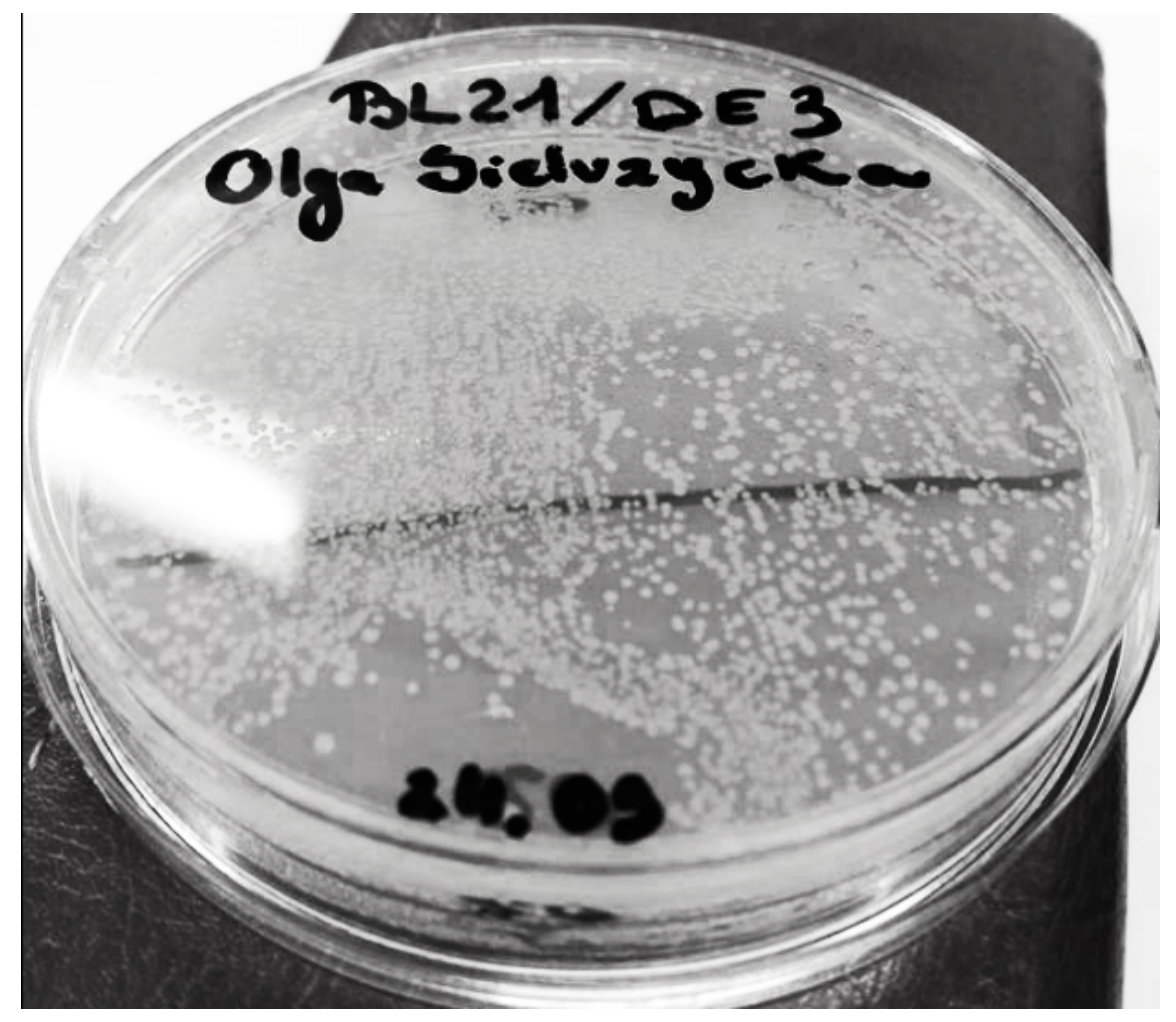

Figure 3: Successfully grown E.Coli culture with a GFP and ampicillin resistance plasmid.

Following the harvesting and re-suspending of the induced cultures the bacteria were purified by running the sample through Nickel charged resin. Both the wash buffer and the elution buffer had constant pH of 8 . This fits in the $\mathrm{pH}$ range in which GFP is stable in being from 6 to 10. (Patterson et al. 1997)

After purification and elution of the GFP containing solution elute was a colorless see-through liquid. It therefore failed to show a presence of GFP that gives elute a bright green color. Furthermore, the SDS gel failed to show a presence of a clear rGFP in the required, $27 \mathrm{~kb}$ elution band. There is a faint band that can be noticed, however it could be just an assembly of unrelated proteins or just not sufficient amount of GFP to consider the expression a success.

Discussion: The aim of the experiment was not only to successfully purify the rGFP but also to appreciate its unique properties and to gain the understanding of common techniques used in modern cloning and expression of recombinant proteins, which was successful as it definitely provided an insight into common methods used in biochemical laboratories.

Obtained results showed a partially successful experiment. The plasmid transformation was successful and sufficient bacterial growth on the antibiotic enriched media was observed as shown by Figure 3A. However 


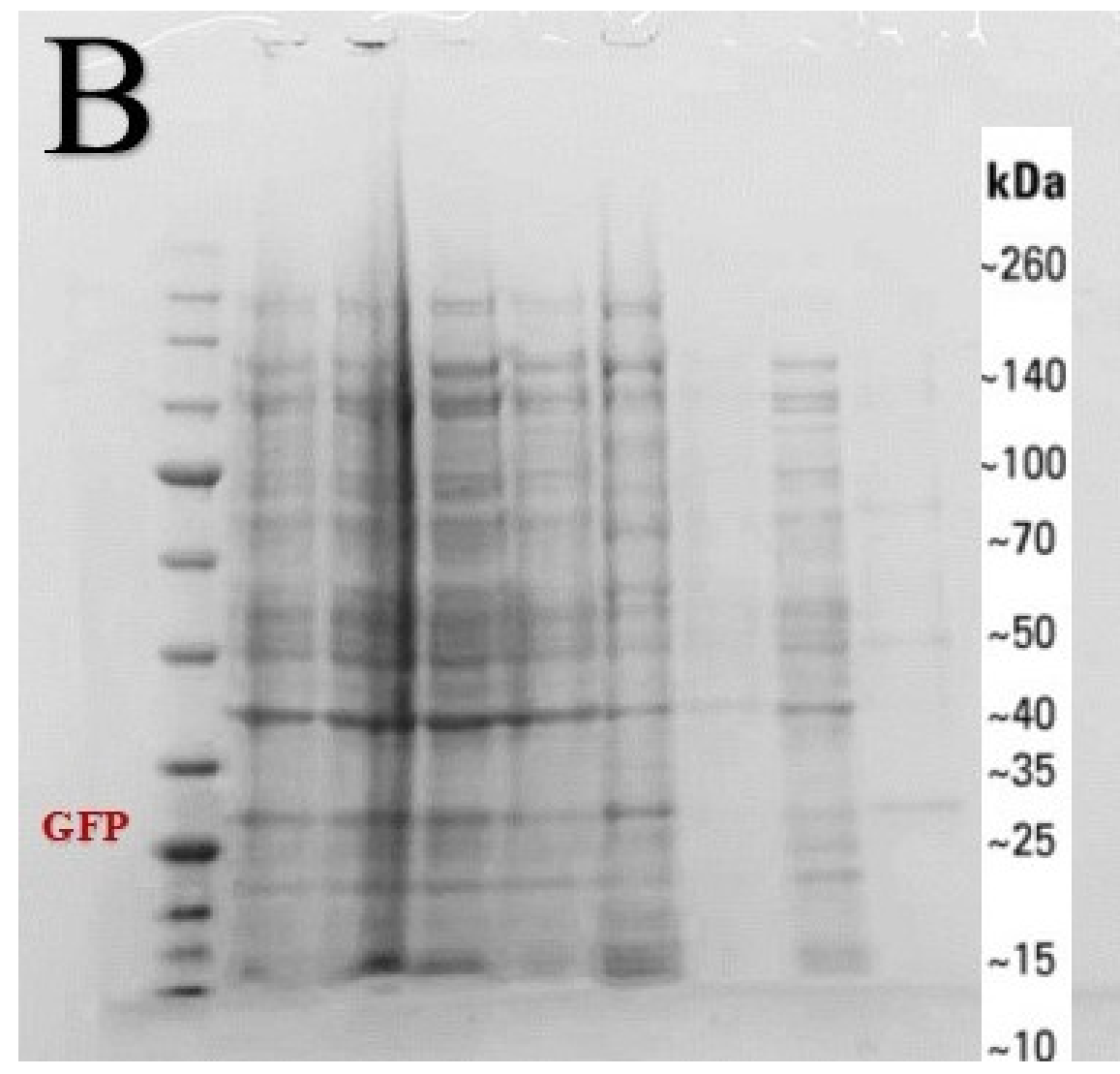

Figure 4: SDS-PAGE visualized by coomassie blue staining with wells containing following samples: 1-ladder, 2-pre induction, 3-post induction, 4-BugBuster, 5-supernatant 6-pellet 7wash 8- flow through 9- elution.

the expression and purification of the protein did not go according to initial assumptions. There are multiple possibilities, why the experiment did not go as planned. While looking at the SDS-PAGE gel, Figure 3B one can notice that the bands in "pre "and "post" induction samples are exactly the same. As mentioned in the "results "section, low amounts of GFP are expressed even without induction due to promoter polymerase overlapping. Similar band widths in "pre and post" induction wells imply that the inoculation did not happen correct and that possibly the amount of IPTG was not sufficient to induce cells. Moreover the induction took place at inappropriate OD and therefore had to be incubated at 370C overnight. This could result in the ampicillin being used up. E.Coli, being incredibly responsive to its environmental changes ejects the plasmid when theantibiotic is not present in its environment. Potentially the prolonged incubation resulted in the antibiotic being used up and the plasmid being ejected and hence no rGFP production was observed. No production theory is further supported by lack of visibly green, rGFP in the re-suspended pellets. Colleagues who managed to obtain rGFP had a clear, bright green suspension. Suspension obtained by myself was brownish yellowish. At this point it was still unclear whether the experiment was successful since the color only did not forejudge the outcome. Despite lack of visibly green solution, there was a good probability that some amounts of rGFP were produced and will become visible after the purification and will be observed after the SDS-page analysis. The IMAC resulted in completely see-through, clear, colorless elute which further indicated that the experiment has failed. Obtained elute should have been a clear, bright green solution due to the rGFP natural properties, and it was observed as such among colleagues whose experiments were successful. Arguably there is a faint bad present on the SDS-PAGE, however I believe that this is nothing more than contaminants. Since the nickel resin is charged, the band is more than likely 
to contain various other proteins that are result of a non-specific binding. To examine the purity of a band further and to fully determine whether the obtained band was a purified rGFP it is essential to perform second chromatographic assay, such as size excursion chromatography. At a current time it is impossible to fully determine the nature of obtained band, it could indeed be a low amount of rGFP but most likely it is a contaminant or a degradation product.

\section{References}

Assets.thermofisher.com. (2018). [online] Available at: https://assets.thermofisher.com/TFS-Assets/LSG/figures/2042.jpg650.jpg.

Dieryck, W., Noubhani, A.M., Coulon, D., Santarelli, X., "Cloning, expression and two-step purification of recombinant His-tag enhanced green fluorescent protein over-expressed in Escherichia coli", J. Chromatogr. B, 786, 153-159(2003)

Jain, S., Teotia, S., Gupta, M.N., "Purification of green fluorescent protein overexpressed by a mutant recombinant Escherichia coli", Protein Expr. Purif., 36, 76-81(2004)

Kumar, Awanish \& Pal, Dr. Dharm. (2016). GREEN FLUORESCENT PROTEIN AND THEIR APPLICATIONS IN ADVANCE RESEARCH 1 2. Journal of Research in Engineering and Applied Sciences. 01. $42-46$.

Patterson, G.H., Knobel, S.M., Sharif, W.D., Kain, S.R., and Piston, D.W.1997. Use of the green fluorescent protein and its mutants in quantitativefluorescence microscopy. Biophys. J. 73: 2782-2790.

Wu, Y., Zhou, Y., Song, J., Hu, X., Ding, Y. and Zhang, Z. (2008). Using green and red fluorescent proteins to teach protein expression, purification, and crystallization. Biochemistry and Molecular Biology Education, $36(1)$, pp.43-54.

ZHOU, X., SHI, Q., XING, X. and SUN, Y. (2006). Rapid Purification of Enhanced Green Fluorescent Protein from Escherichia coli. Chinese Journal of Chemical Engineering, 14(2), pp.229-234. 